TITRE: LES PROJETS CLINIQUES À LA FRONTIÈRE DE LA RECHERCHE EXPÉRIMENTALE ET DU TRAITEMENT INNOVATEUR: QUEL CONSENTEMENT?

Auteur(s): Johane PATENAUde

PublicATION: RECRUTEMENT ET CONSENTEMENT À LA RECHERCHE : RÉALITÉS ET DÉFIS ÉTHIQUES

PAGES: $39-53$

ISBN: 978-2-7622-0357-8

Directeurs: Ana Marin, BÉATRICE Eysermann et Michel T. GIROUX

URI: HTTP://HDL.HANDLE.NET/11143/14106

DOI: HTTPS://DOI.ORG/10.17118/11143/14106 


\section{Les projets cliniques à la frontière de la recherche expérimentale et du traitement innovateur : Quel consentement?}

Johane Patenaude, professeure titulaire et chercheure, Département de chirurgie, Faculté de médecine et des sciences de la santé, Université de Sherbrooke

Résumé : Le consentement à un traitement expérimental (activité de recherche) exige la prise en compte d'informations plus exhaustives que celles exigées pour consentir à un traitement innovateur (activité clinique). D'où l'importance de déterminer adéquatement le statut auquel correspond effectivement le traitement proposé. Or les définitions visant à départager la recherche de l'innovation sont reconnues depuis longtemps pour être peu opérationnelles, entraînant des risques de confusion. Cette confusion possible a un impact direct sur le consentement, puisque la nature du consentement requis dépend du statut qu'on accorde à l'intervention concernée.

Ce problème est décrié depuis plus de 15 ans par de nombreux chercheurs, cliniciens, gestionnaires et autres parties prenantes. Où en sommes-nous aujourd'hui? Pratiquement au même point. C'est ce dont il sera question ici. À partir des principaux jalons de l'itinéraire de la notion de traitement innovateur depuis sa survenue dans les documents normatifs québécois, nous dégagerons quelques lieux de confusion quant à son opérationnalisation en recherche et en clinique. Finalement, quelques pistes de solution seront proposées.

Mots clés : Éthique, soins innovateurs, évaluation éthique de l’innovation, consentement.

Abstract: Consent to an experimental treatment calls for a more exhaustive consideration of the relevant information than that is required to agree to an innovative treatment (clinical activity). It is thus important to adequately determine the status to which the proposed treatment effectively corresponds. However, the definitions aimed at defining the difference between research and innovation have been recognized to be minimally operational for a long time, which is causing some risks of confusion. Such possible confusion has a direct impact on consent, since the very nature of the required consent depends on the status given to the intervention at stake.

This problem has been decried by numerous researchers, clinicians, managers and other stakeholders for more than 15 years. Where are we at today? Practically at the same point. This is what will be discussed here. Starting with the principal itinerary milestones of the notion of innovative treatment presented at the beginning of its occurrence in normative Quebec documents, we will extract statements where the confusion has been documented with respect to the operationalization aspect in research as well as in the clinical field. Finally, a few paths of solutions will be proposed.

Keywords: Ethics, innovative care, ethical evaluation of innovation, consent 


\section{Introduction}

Les années 90 donnent lieu à un foisonnement d'écrits et de communications en éthique de la recherche, notamment dans le cadre de la recherche biomédicale. La médecine occidentale contemporaine privilégiait déjà les connaissances fondées sur des données probantes (Sackett, 1997 : 3-5). Parallèlement, c'est sur cette base que les écrits de l'époque relancent l'importance de la recherche biomédicale scientifique en vue de nouvelles interventions diagnostiques et thérapeutiques au grand bénéfice des patients (Wilfond, 1995). Bien que jugées nécessaires, ces activités de recherche issues de données probantes soulevaient déjà des critiques au plan éthique en raison des risques encourus pour le patient et du besoin de rappeler que ces activités ne peuvent se faire aux dépens de valeurs éthiques fondamentales de notre société (Kerridge et coll., 1998; Tonelli, 1998), dont celles liées au respect de l'autonomie et qui se traduisent de manière privilégiée dans l'obtention d'un consentement libre et éclairé. Le défi posé par la société dans le continuum des activités de recherche, de l'innovation et de l'exercice de la médecine devenait celui d'établir les limites de l'acceptable (Desjardins, 1997).

Au Canada comme dans de nombreux pays, lorsqu'une nouvelle intervention médicale est utilisée sur une base expérimentale chez des sujets humains, on exige qu'un protocole applicable à ces sujets soit d'abord soumis à l'examen d'un comité d'éthique de la recherche (CER) pour approbation. Le contrôle des CER ne s'exerce plus à partir du moment où un traitement a dépassé l'étape expérimentale; ce dernier relèvera dès lors de la pratique clinique. Il existe toutefois une zone d'ombre entre le statut expérimental d'un traitement, considéré comme de la recherche et encadré comme tel par les CER, et le statut d'un traitement pleinement reconnu en clinique. Cette zone d'ombre, c'est le traitement ou soin dit «innovateur». Ce type de soins ne s'inscrit pas dans un contexte de recherche et vise plutôt l'intérêt d'un patient. Selon Avard et coll. (2004), se référant à Deleury (2002 : 145-147) : «lls consistent en une initiative spontanée d'offrir un traitement inédit, non validé, non éprouvé et non approuvé par les pairs». Par exemple, «[l]es thérapies nouvelles peuvent être des techniques

d'intervention chirurgicale, l'utilisation de nouveaux appareils d'investigation ou de traitement, des greffes d’organes, l'administration de médicaments, etc.» (Kouri et Philips-Nootens, 1996-97 : 108).

Ce statut intermédiaire, nous le verrons, soulève d'importants défis sur plusieurs plans, notamment au regard des informations requises à transmettre aux éventuels patients à qui ces traitements innovateurs seraient proposés dans le cadre de leurs soins, de leur compréhension de celles-ci et conséquemment au regard du respect du caractère libre et éclairé de leur consentement.

En effet, puisqu'un traitement qualifié d'innovateur n'est pas soumis à une évaluation éthique comme c'est le cas en recherche (celui-ci relevant plutôt de la pratique clinique) la nature et la portée du consentement du participant pourraient alors être très différentes selon la qualification donnée à la procédure, expérimentale ou innovatrice. Plusieurs projets impliquant de nouveaux soins font en sorte que la question se pose encore aujourd'hui de savoir s'ils relèvent de l'expérimentation sur l'humain ou s'ils s'inscrivent plutôt parmi les pratiques cliniques. De fait, la mince frontière qui sépare l'expérimentation et l'innovation, dans le continuum de la recherche et la clinique, peut occasionner des difficultés pour les CER, les cliniciens ou toute autre partie prenante ayant à déterminer si une procédure relève ou non de leur champ de compétences. 
Les professionnels de la santé, et particulièrement les médecins, peuvent également rencontrer des difficultés à déterminer dans quelle mesure leurs activités relèvent des soins cliniques ou de la recherche sur l'humain. En effet, la recherche clinique et l'exercice de la médecine ne s'effectuent généralement pas dans des milieux étanches; ils cohabitent souvent dans un même établissement de santé et par les mêmes intervenants qui cumulent les rôles de clinicien et de chercheur. Ce rapprochement entre les activités de recherche et de soins pourrait augmenter la confusion à l'égard de la finalité des procédures : faire avancer les connaissances ou soigner (Edgar and Rothman, 1995)?

L'un des effets néfastes de cette confusion serait de laisser libre cours à des traitements expérimentaux sur des patients en clinique sous le couvert de soins estimés innovateurs, voire approuvés. En conséquence, l'encadrement éthique et scientifique requis pour de telles activités de recherche clinique pourrait s'en trouver sensiblement réduit et ainsi exposer les patients à des risques indus ou auxquels ils n'auraient pas consenti librement ou en toute connaissance de cause. Cette préoccupation génère la question suivante : À quel moment les nouvelles interventions en clinique devraient-elles s'astreindre aux mêmes normes de protection des sujets que celles qui s'imposent aux chercheurs (Moutel et coll., 1998)? Répondre à cette question implique la capacité des décideurs de maîtriser les caractéristiques propres aux différents types d'activités qui jalonnent, tels un continuum, la recherche biomédicale et l'exercice de la médecine, ainsi que les dispositifs normatifs propres à chacun de ces types d'activités comme peut l'être, par exemple, le Comité d'éthique de la recherche dans le cas d'une activité de recherche expérimentale impliquant le concours de sujets humains. D'autres questions sollicitent ces compétences, comme celle de déterminer à quel moment la modification d'une technique standardisée représente-t-elle plus qu'une modification et devient-elle, du coup, un traitement expérimental, c'est-à-dire de la recherche (Patenaude et Cabanac, 2000).

Ces enjeux, loin d'être nouveaux (Moore, 1969; Blancquaert, 2000; Blancquaert et coll., 2001), sont plus que jamais d'actualité en raison de plusieurs facteurs, dont le délai de plus en plus court exigé entre le développement des connaissances issues de la recherche fondamentale et leurs possibles applications (sur le marché?) en clinique par les organismes subventionnaires, les promoteurs, voire les centres de recherche et les universités elles-mêmes. Cette rapidité hautement valorisée pourrait contribuer à intensifier la tentation néfaste que soulevaient déjà Kouri et Philips-Nootens en 1997 (103):

La tentation pourrait être grande, pour certains, de qualifier un acte de «soin innovateur» plutôt que de «thérapie expérimentale», car il serait alors possible pour les chercheurs de procéder sans contrainte externe. Il y aurait ainsi rétrécissement ou compression de la phase expérimentale permettant d'aboutir plus rapidement à la phase innovatrice, peut-être au détriment de la sécurité des personnes.

Ces auteurs poursuivent, soulignant à juste titre la teneur d'ordre éthique - plutôt que juridique - de ces enjeux:

Des situations factuelles très similaires peuvent ainsi mener à des qualifications différentes. Est-il vraiment opportun d'attendre le développement d'une «jurisprudence éthique» sur la question afin de pouvoir préciser l'acception exacte des expressions utilisées par le législateur? 
Si les soins innovateurs génèrent beaucoup de méfiance et d'inquiétude, particulièrement chez les bioéthiciens, juristes et cliniciens en Amérique du Nord (Kouri et Philips-Nootens, 1996-97; Agich, 2001; Blancquaert et coll., 2001; Patenaude et Cabanac, 2000; Kouri, 2013) et en France (Moutel et coll., 1998), ceux-ci n'en reconnaissent pas moins les avantages indéniables et la pertinence. Là n'est pas la question, on l'aura compris. Du reste, il convient aussi de noter que la situation inverse, c'est-à-dire de qualifier d'expérimental un traitement qui serait bien plutôt innovateur, serait tout autant susceptible d'engendrer des préjudices envers les patients à qui ces soins innovateurs sont destinés, ne serait-ce qu'en raison des délais indus impliqués pour l'obtention de l'approbation du CER et la préparation conséquente du dossier exigé à cette fin par le clinicien.

Agich (2001 : 296) résume bien cet enjeu. Selon lui, la demande d'un essai clinique dans un domaine de la médecine en cours de développement rapide et dynamique peut entraver l'innovation. Dans certaines circonstances, écrit-il, le retard dans le développement et l'adoption de tout nouveau traitement est un prix à payer. Chaque fois qu'il existe d'autres traitements standards avec un degré de succès acceptable et qui ne sont pas lourds, les essais cliniques sont justifiés. Cependant, poursuit-il, il est moins clair que les retards soient éthiquement justifiés dans les domaines de la médecine ou des maladies où il n'y a pas de traitements satisfaisants ou lorsque les traitements sont encombrants ou problématiques. Agich reconnaît néanmoins du même souffle que les critères aussi vagues que lourds ou problématiques sont, bien sûr, empreints de controverses et de matière à débat (2001 : 296).

Qu'en est-il de ces enjeux aujourd'hui, sommes-nous mieux outillés pour y faire face? Où en sommes-nous? Un tel bilan suppose de revenir sur quelques-uns des principaux concepts proposés dès le début des années 90 par les instances normatives québécoises et dont les définitions visaient justement à faciliter la détermination des procédures concernées dans un souci de respect de l'autonomie des personnes, notamment des personnes vulnérables, en assurant un plein consentement à l'intervention qui leur serait proposée dans le cadre de leurs soins ${ }^{1}$. Il n'est donc pas étonnant que le souci de définir ces concepts se soit manifesté par les instances normatives visant à encadrer les activités de recherche, dont l'expérimentation sur l'humain fait partie. Voyons-les brièvement.

\section{Quelques concepts et définitions}

Le Ministère de la Santé et des Services sociaux (MSSS) du Québec proposait, en 1998, les trois définitions suivantes dans son Plan d'action ministériel (PAM) en éthique de la recherche et en intégrité scientifique (MSSS, $1999: 25-26)$ :

Acceptée: Une procédure dont l'efficacité clinique, les indications et les protocoles sont bien établis sera désignée comme acceptée.

Il s'agit de soins standards pour lesquels le consentement aux soins est requis. Le CER n'est pas requis.

\footnotetext{
1. Bien qu'il s'agisse d'une revue des définitions dans le contexte québécois, il convient de noter que ces définitions, dans un contexte de recherche français, ont démontré la même confusion chez les décideurs, ainsi que les mêmes limitations conséquentes au regard des informations à transmettre aux patients participants à ces traitements à la frontière de l'expérimentation et du traitement innovateur (Moutel et coll.,1998; Patenaude et Cabanac, 2000). Nous ignorons malheureusement ce qu'il advient aujourd'hui de cette situation en France. Notre bilan se bornera ici à l'évolution de cette problématique au Québec, ainsi qu'ailleurs au Canada en raison du partage de plusieurs cadres normatifs en recherche en matière de consentement.
} 
Expérimentale : Par contre, une procédure dont l'efficacité clinique n’a pas encore été reconnue est désignée comme expérimentale. Nous ne savons pas si elle produit les bénéfices escomptés. Donc, on ne s'attend pas à ce qu'une telle procédure soit acceptée par les services de santé, sauf dans un protocole de recherche.

Le consentement à la recherche est requis par la signature d'un formulaire détaillé sur l'objet du projet, les implications à y prendre part, etc. Le CER assure ainsi l'évaluation éthique et le suivi du projet.

Innovatrice: Il reste des procédures qui ont dépassé l'étape expérimentale. Leur efficacité a été établie, mais, vu le manque d'expérience, les modalités d'application et même les indications exactes pour ces interventions sont à préciser. On appelle une telle technologie innovatrice [...].

Les procédures qualifiées d'innovatrices ne requièrent pas de consentement à partir d'informations détaillées comme dans le cas de la procédure expérimentale 2 . Cette définition de la notion de procédure innovatrice du PAM, mentionnée ci-dessus, provient du Conseil d'évaluation des technologies de la santé (CETS, 1991 : 2-3) qui la proposait en 1991 à l'occasion d'un rapport sur les transplantations d'organes au Québec. Selon le PAM, cette définition, qu'il juge limitée, aurait toutefois «permis d'atteindre, dans des domaines similaires, un consensus important tout en conservant la flexibilité nécessaire pour s'adapter au développement de la recherche» (MSSS, 1998 : 25). Or, rien n'est moins sûr, comme on le verra dans la partie qui suit.

\section{Procédure innovatrice ou expérimentale : quels repères, selon qui?}

Tenter de définir rigoureusement et de manière opérationnelle la notion de procédure innovatrice fut sans doute une épine de taille dans la chair du MSSS, notamment au regard de la rédaction de son PAM en éthique de la recherche et en intégrité scientifique. Ce passage du PAM (MSSS, 1998 : 25) en témoigne de lui-même:

Pendant la période de consultation de la commission parlementaire chargée d'examiner l'avant-projet de loi modifiant le Code civil en matière de recherche biomédicale, plusieurs organismes ont mentionné la difficulté qu'ils avaient d'arriver à une définition entièrement satisfaisante du concept de soins innovateurs. Ce constat est important puisque définir une activité comme s'il s’agissait de soins innovateurs plutôt que d'une activité de recherche soustrait par la suite celle-ci au mécanisme d'examen éthique, alors que c'est le comité d'éthique de la recherche qui détermine ce statut.

Dans le cas où il appartient au comité d'éthique de la recherche (CER) d'accorder un tel statut, il n'existe cependant pas d'exigences explicites quant à la nature et à la portée de l'information qui doit lui être soumise pour ce faire. On peut alors se questionner sur les critères mobilisés par les CER pour procéder à cette évaluation (la littérature n'en rend pas compte). De plus, on ignore si ces évaluations sont partagées d'une instance à l'autre ou si, au contraire, elles sont divergentes.

Ces questions furent à la base de l'une de nos études empiriques, qui a clairement démontré la confusion tant chez les 27 CER que chez les 37 cliniciens biochimistes à qui l'on demandait de qualifier le type de procédure concerné face à un même projet sur la base des trois définitions du MSSS énoncées précédemment (Patenaude et coll., 2008). Sans revenir sur les détails de cette enquête pancanadienne, il convient néanmoins d'en

\footnotetext{
2. Dans le cas d'activités jugées innovatrices, le PAM précise dans la définition quelques consignes sur leur portée générale ainsi que sur leur cadre global de réalisation: «Afin d’augmenter le niveau de connaissance, il est important de recueillir toute expérience future des applications de cette technologie de façon systématique et de communiquer ces expériences au monde médical. Pour cette raison ces activités ne doivent se poursuivre que dans une institution universitaire autorisée où les ressources requises sont disponibles » (MSSS, $1999: 26)$.
} 
reprendre ci-dessous les résultats obtenus pour trois de ses questions. Les résultats surlignés dans les tableaux qui suivent méritent attention.

Question

Selon-vous, est-ce que ce projet constitue de la recherche?

\begin{tabular}{|c|c|c|c|}
\hline & Biochimistes & CÉR & N \\
\hline Oui & 30 & 14 & 44 \\
\hline Non & 7 & 13 & 20 \\
\hline $\mathbf{N}$ & 37 & 27 & 64 \\
\hline
\end{tabular}

Question

Selon-vous, quelle procédure fait l'objet de ce projet?

\begin{tabular}{|l|c|c|c|}
\hline & Biochimistes & CÉR & N \\
\hline Procédure expérimentale & 3 & 2 & 5 \\
\hline Procédure innovatrice & 25 & 10 & 35 \\
\hline Procédure acceptée & 4 & 5 & 9 \\
\hline Autre & 5 & 9 & 14 \\
\hline $\mathbf{N} \backslash \mathbf{R}$ & 0 & 1 & 1 \\
\hline $\mathbf{N}$ & 37 & 27 & 64 \\
\hline
\end{tabular}

\begin{tabular}{|l|c|c|c|}
\hline \multicolumn{3}{|c|}{ Question } \\
\hline \multicolumn{3}{|c|}{ Selon vous, quelle procédure fait l'objet de ce projet? } \\
\hline CÉR & Biochimistes & CÉR & N \\
\hline CÉC (comité éthique clinique) & 16 & 12 & 28 \\
\hline Investigateurs & 8 & 2 & 10 \\
\hline DSP ou CMDP & 7 & 2 & 9 \\
\hline CÉR et CÉC & 0 & 8 & 8 \\
\hline Investigateurs et cliniciens impliqués & 3 & 0 & 3 \\
\hline Autres & 1 & 0 & 1 \\
\hline N/R & 1 & 3 & 4 \\
\hline N & 1 & 0 & 1 \\
\hline
\end{tabular}

Comme le décriait un groupe d'experts de l'Agence en évaluation des technologies et des modes d'intervention en santé du Québec (AETMIS-INESSS) : «The collection of validity and utility data is essential not only to further scientific knowledge but also to be able to adequately inform affected individuals» (Blancquaert et coll., 2001).

Il importe donc plus que jamais d'identifier les lieux de confusion possibles dans la compréhension, l'application et l'opérationnalisation des distinctions entre les types de procédures. De nombreux travaux théoriques issus des années 90 ont porté sur l'importance de mieux définir cette zone d'ombre incontournable qu'est le soin innovateur dans le continuum liant la recherche aux interventions approuvées en clinique (Prentice et coll., 1997; Fost, 1998; Lind, 1992; Edgar et Rothman, 1995; ACP-ASIM, 1998, et de nombreux autres). Un tel foisonnement convergeant dans la littérature n'est pas le fait du hasard; cette décennie fut le théâtre de l'apparition massive de plusieurs technologies dites «de rupture» en santé (génétique, génomique, clonage 
animal/humain, imagerie, neurosciences, nanotechnologies, implants de tout ordre, incorporation d'organismes génétiquement modifiés, néonatalogie, etc.). Un espace plus considérable que jamais émergeait alors entre le laboratoire et le cabinet du médecin. Cet espace, qui n’est ni recherche ni soin standardisé, restait donc à circonscrire.

Au Québec, le Conseil d'évaluation des technologies de la santé (CETS, 1991) situait l'innovation dans le chevauchement des procédures acceptées et celles dites expérimentales. Cette définition, nous l'avons vu, fut reprise telle quelle par le MSSS en 1998. Cette définition s'inscrivait dans la foulée de l'article 21 du Code civil du Québec (CCQ), qui, jusqu'en 2013, précisait : «Ne constituent pas des expérimentations les soins qui, selon le comité d'éthique, sont des soins innovateurs requis par l'état de santé de la personne qui y est soumise.» En apparence clair, cet article 21 énonçant certaines règles concernant l'expérimentation médicale demeurait une source de confusion tant pour les CER que pour les investigateurs et les cliniciens, car il vise à la fois la réglementation des soins expérimentaux, de l'expérimentation purement scientifique et des soins innovateurs, sans toutefois définir ni préciser la portée de ces termes (Kouri et Philips-Nootens, 1996-97; Weisstub et Verdun-Jones, 1997).

Procédure innovatrice, soin ou traitement innovateur... Ce travail de définition, bien que très complexe, visait alors à répondre à un besoin. Qu'en est-il de ce besoin aujourd'hui et de quels moyens disposons-nous collectivement pour y répondre?

\section{Où en sommes-nous?}

Ce travail de définition de procédures (acceptées, expérimentales ou innovatrice) visait, dès le début des années 90, à répondre au besoin d'encadrer les procédures innovatrices en les distinguant des autres pour assurer dans ce cas l'obtention d'un consentement conséquent de patients ou participants éventuels. Le PAM ciblait clairement ce besoin en 1998, en précisant (26) : «Afin de guider les comités d'éthique de la recherche et de les doter de balises communes s'agissant de la notion de soins innovateurs - le ministère n'y était pas encore parvenu efficacement - , le Ministère de la Santé et des Services sociaux du Québec confie au FRSQ³, au CQRS ${ }^{4}$, au Collège des médecins du Québec ainsi qu'au CETS le mandat de préciser davantage le concept de soins innovateurs dans le cadre de l'application de l'article 21 du Code civil». Un appel à tous fut ainsi lancé.

Un long silence s'ensuivit. On ne s'étonne donc pas que Sonia Audy, mandatée pour revoir l'ensemble de la politique ministérielle (PAM), n'ait pu consacrer plus qu'une quinzaine de lignes dans son rapport sur cette question. On ne s'étonne pas non plus du titre choisi pour cette section du rapport, «Les soins innovateurs : un dossier dont personne ne veut...» (Audy, 2006 : 253).

Il faudra attendre les années 2008-2009, soit 10 ans, pour y trouver écho. C'est le FRSQ (aujourd'hui le FRQS) qui donna suite à cet appel dans son document intitulé Les standards du FRSQ sur l'éthique de la recherche en santé humaine et l'intégrité scientifique. Cet important document y consacrait une section d'une demi-page intitulée «L'expérimentation et les soins innovateurs» (FRSQ, 2008, Section 25 : 41), essentiellement calquée sur le CCQ:

3. FRSQ : Fonds de recherche en santé du Québec, devenu aujourd'hui le Fonds de recherche du Québec en santé (FRQ-S).

4. CQRS : Conseil québécois pour la recherche sociale 
L'article 21, al. 4 du Code civil du Québec traite les expérimentations et les soins innovateurs comme étant des interventions distinctes: «Ne constituent pas des expérimentations les soins qui, selon le comité d'éthique, sont des soins innovateurs requis par la personne qui y est soumise.»

L'importance de cette distinction tient au fait que les soins innovateurs peuvent être dispensés suivant les normes juridiques applicables aux soins requis par l'état de santé plutôt que d'après les normes qui concernent l'expérimentation.

Le Code civil ne définit pas ce que sont les soins innovateurs et l'expérimentation. Pour le moment, il revient au CER d'identifier ce qui caractérise chacun de ces deux types d'interventions lorsque cette distinction doit être faite.

Le FRSQ fut sans doute conscient du fait que son propos n'ajoutait pas d'eau au moulin, car il termine ainsi : "Le FRSQ produira un document qui interprète et clarifie les trois concepts suivants, que contient l'article 21 du Code civil : "projet de recherche", "expérimentation" et "soins innovateurs"».

Ce projet n'a pas eu de suite; il semble que le FRSQ (ou FRQS) ne produira pas ce document interprétant et clarifiant les concepts qui figuraient au quatrième et dernier alinéa du CCQ. En effet, parmi les modifications apportées au Code civil du Québec en juin 2013, on remarque que cet alinéa, qui portait sur les soins innovateurs, a été supprimé. Conséquemment, il ne revient plus aux CER de déterminer le statut, expérimental ou innovateur, d'une procédure. Les procédures innovatrices relevant juridiquement des soins dans le CCQ. depuis 2013, il revient donc désormais au clinicien, et non au CER, de déterminer ce statut. Si cette nouvelle posture du CCQ compense certaines faiblesses au plan juridique ${ }^{5}$, la zone d'ombre demeure entière au plan éthique. Comme le soulève Viens (2015: 16):

Tel qu'établi jusqu'en 2013 par le libellé de l'article 21 C.c.Q., le CER possédait une grande responsabilité quant à la détermination de la nature d'un acte thérapeutique donné. Lorsque ce dernier était considéré comme un soin innovateur requis par l'état de santé de l'individu, il était incertain si les règles relatives aux soins courants ou celles applicables à la recherche s'appliquaient. Par opposition, lorsque le comité d'éthique concluait à la recherche à visée thérapeutique, la protection de l'article 21 C.c.Q. était assurée. Maintenant que l'exception relative aux soins innovateurs n'est plus au Code civil, l'incertitude demeure tout de même quant aux règles applicables à ces derniers. Les soins innovateurs sont-ils assujettis aux règles relatives aux soins courants ou à la recherche?

Le problème, on le voit, reste entier au plan éthique : quel statut, donc quel consentement?

\section{Pistes de solution}

La littérature médicale internationale abonde de pistes de solution, au moins théoriquement, particulièrement dans les spécialités chirurgicales (Broekman et coll., 2016). Malheureusement, il existe peu ou pas de littérature ou de rapport rendant compte de leur mise en œuvre effective dans les milieux de pratique. La situation ne semble pas plus simple du côté de la gouvernance, comme en témoigne l'initiative d'un récent projet de loi au Royaume-Uni visant à encadrer les cliniciens dans la détermination du statut, innovateur ou non, accordé à une procédure innovatrice envisagée pour leurs patients. Selon Kouri (2013, note p. 875) :

5. Kouri (2013: 875$)$ précise : «Plus fondamentalement cependant, cet alinéa comportait une faiblesse évidente : comment un comité d'éthique pourrait-il déterminer d'avance si des soins sont innovateurs ou non ? Logiquement, une telle détermination en serait une de nature strictement scientifique. Qu'il s'agisse d'innovation ou d'expérimentation, dans un cas comme dans l'autre, l'on se retrouve dans le domaine de la recherche et l'on doit, de toute façon, se conformer aux exigences du Code civil ». 
[...] c'est en partie pour cette raison que le projet de loi intitulé «Medical Innovation Bill (N²), 2013-14 (Bill 107)», fut présenté en première lecture à la Chambre des Communes du Royaume (Parliament UK, 2013). On y proposait que tout soin innovateur doit être approuvé par une équipe multidisciplinaire de médecins («multidisciplinary team of doctors»).

Ce projet de loi a cependant été retiré et ne progressera plus, et le site internet dédié n'en donne malheureusement pas la raison. Une telle piste d'évaluation et de décision en comité de pairs semble néanmoins prometteuse si, bien sûr, les membres disposent d'un cadre de référence et de critères clairs et partagés pour guider leur jugement dans l'exercice de ce que les philosophes éthiciens nomment la pensée pratique. À défaut, nous pourrions voir se répéter des résultats semblables à ceux de notre enquête pancanadienne et dont nous faisions sommairement état plus tôt.

Des propositions de solutions furent aussi présentées dans le cadre de la psychopharmacologie. Le titre du principal article dans le domaine parle de lui-même : «The ethics of clinical innovation in psychopharmacology: Challenging traditional bioethics» (Ghaemi et Goodwin, 2007). À l'instar de plusieurs cliniciens, dont Agich (2001), les auteurs insistent sur le manque de reconnaissance des bioéthiciens à l'égard de la spécificité de cette zone d’ombre que sont les traitements innovateurs. Néanmoins conscients des enjeux éthiques liés à cette phase intermédiaire, ces auteurs déplorent les limites de la bioéthique traditionnelle - et, du coup, celle des bioéthiciens qui s’y réfèrent - pour répondre aux enjeux éthiques qui y sont soulevés. Selon Ghaemi et Goodwin (2007) (c'est nous qui traduisons) :

Une évaluation conceptuelle et historique de ce sujet souligne l'importance éthique et scientifique de l'innovation clinique. Éthiquement, cela touche une limite qui, à notre avis, n'est pas adéquatement encadrée dans la bioéthique traditionnelle contemporaine. À l'heure actuelle, la recherche est considérée comme ne bénéficiant pas du tout aux patients qui y participent, tandis que les soins cliniques sont considérés comme étant uniquement pour le bénéfice des patients. L'innovation clinique chevauche ces deux mondes, parfois malaisément. Bien que plusieurs soutiennent que l'innovation clinique doit être évitée ou s'inscrire dans des projets de recherche, nous soutenons que l'innovation clinique est nécessaire pour progresser dans la recherche en psychopharmacologie et qu'elle pourrait mieux prospérer mieux si elle était guidée par les principes éthiques suivants : 1) le traitement devrait porter sur la base d'une hypothèse viable; 2) dans la mesure du possible, les observations cliniques devraient être signalées afin qu'elles puissent être évaluées par la communauté scientifique; 3) on devrait être disposés à signaler des observations inattendues des effets sur les médicaments; 4) un niveau élevé de consentement éclairé devrait être maintenu.

Ghaemi et Goodwin (2007) insistent pour dire que cette proposition va à l'encontre de la vision qui prévaut chez les bioéthiciens et selon laquelle la recherche et les soins cliniques sont des activités catégoriquement opposées, comme l'explique l'un ou l'autre ou la dichotomie du rapport Belmont sur la bioéthique. En effet, selon ces auteurs, cette approche a tellement polarisé leur profession en tant que cliniciens par rapport aux chercheurs, que de nombreux cliniciens n'appliqueront pas les nouvelles connaissances produites par la recherche clinique jusqu'à ce qu'elle soit incorporée dans les lignes directrices formelles de traitement, alors que, d'autre part, les chercheurs ont peu d'éléments pour les guider au regard du type de nouvelles connaissances les plus importantes à fournir.

Cette proposition «hybride» de Ghaemi et Goodwin (2007), empruntant tant à la recherche qu'à la clinique, trouve un certain écho chez plusieurs auteurs, dont Blancquaert et coll. (2001 : 106-107). Pour ces derniers, une fois que le statut des soins innovants est accordé, le manque de suivi scientifique et éthique qui en résulte 
pourrait avoir de graves conséquences. Compte tenu des incertitudes entourant les soins innovants, le suivi des patients se soumettant à ces traitements devient essentiel. Cette période de suivi doit, selon eux, être utilisée pour en mesurer l'impact et pour mettre à jour les données requises pour un consentement éclairé. En effet, selon Blancquaert et coll. (2001), seule une boucle d'information récursive peut assurer un véritable consentement éclairé aux essais suite à la phase de transition. La proposition de Blancquaert et coll. (2001) semble cependant plus engageante au plan éthique que celle de Ghaemi et Goodwin (2007), en soutenant que le concept de soins innovants, par opposition à l'expérimentation, ne devrait être invoqué que si un suivi scientifique et éthique est assuré.

Si ces pistes de solutions sont intéressantes pour plusieurs, d'autres les jugent contre-productives, dont Agich (2001 : 296), qui estime que le problème est plus profond et qu'il ne saurait être résolu par de telles pistes hybrides. Selon lui, dans le soin innovant, on a recours à des protocoles cliniques traitant des préoccupations cliniques pratiques et des paramètres finaux plutôt qu'à des hypothèses ou des méthodes scientifiques. SeIon lui, ces protocoles articulent les objectifs cliniques et intègrent les processus complexes de découverte et d'expérience acquise avec une procédure de développement. Ils peuvent, dit-il, être remarquablement différents des protocoles scientifiques qui découlent d'une déclaration claire d'une question de recherche sous les contraintes relativement rigides de la méthodologie scientifique. L'écart entre les postures précédentes et celle d'Agich (2001) semble se creuser davantage quand il insiste à l'effet que l'innovation tolère, et prospère même, sur la base de l'intuition, de l'expérience et de l'incertitude, alors que la recherche scientifique se déroule sous les contraintes méthodologiques d'une base de connaissance établie vers son but de confirmation ou de rejet d'hypothèses. Ainsi, il semblerait que l'écart entre recherche clinique et innovation clinique qu'indique Agich (2001) soit d'ordre épistémologique.

Pour notre part, il semble clair qu'une solution à ces enjeux ne peut être que le fruit d'une collaboration forte et soutenue entre les diverses parties prenantes, par exemple au sein d'un comité d'éthique clinique de l'innovation (CECI) qui serait reconnu par les instances des établissements de santé et les institutions universitaires et qui serait composé d'acteurs issus de la recherche (centre de recherche hospitalier ainsi qu'universitaire, le cas échéant), de la pratique clinique, de la gestion des soins, d'associations des usagers et de toute autre partie prenante. La participation active à ce comité de l'Unité d'évaluation des technologies et des modes d'intervention en santé et services sociaux (UETMISS) de ces établissements ${ }^{6}$ serait également souhaitable. La seule mise en place d'une telle instance ne serait évidemment pas une garantie de succès; les compétences et l'expérience de chacun devront être mobilisées dans une entreprise de coapprentissages et de valeurs partagées au service d'une innovation responsable dans nos institutions.

La tâche est plus que jamais complexe dans le contexte où les collaborations de recherche université-industrie ont été essentielles à un haut degré d’innovation technologique en médecine. Récemment, cependant, des questions critiques ont été posées sur les aspects négatifs potentiels des moyens hautement productifs d'encourager l'innovation. Les préoccupations portent sur les rôles flous entre la recherche académique et le monde commercial et les implications de la volonté nouvelle des universités de bénéficier financièrement de leur propriété intellectuelle. Comme le soulèvent Gelijns et Thier (2002), les rôles des deux parties sont souvent insuffisamment pris en compte en considérant que les membres des facultés universitaires sont consacrés à l'avancement des connaissances fondamentales et des entreprises industrielles en tant que simples déve-

6. L'Unité d'évaluation des technologies et des modes d'intervention en santé et services sociaux (UÉTMISSS) aide les décideurs des établissements de santé en réalisant, en soutenant et en encadrant des projets d'évaluation de technologies et de façons de faire en santé et services sociaux. L'UETMISS évalue ou encadre tout projet d'évaluation qui touche les équipements de haute technologie, la dispensation et l'organisation des soins, les nouvelles pratiques, les interventions médicales et chirurgicales, les équipements, les systèmes de soutien, les diagnostics, les médicaments, etc. 
loppeurs de recherche universitaire. Au contraire, l'innovation médicale dépend d'interactions approfondies entre les universités et l'industrie, les connaissances et le transfert de technologie dans les deux sens. Ces interactions ont eu d'importants avantages pour la santé publique et l'économie.

Pourtant, il existe un risque pour la relation université-industrie si les principes culturels et éthiques d'un partenaire maîtrisent ceux de l'autre. Par conséquent, les universités, les centres de recherche hospitaliers et l'industrie doivent maximiser les retombées de la collaboration par un encadrement socialement responsable et minimiser les inconvénients au moyen d'un changement organisationnel interne.

Oeuvrant, chemin faisant, au cœur de ces enjeux complexes, un comité d'éthique clinique de l'innovation (CECI), multidisciplinaire et multicomposite par essence, ferait œuvre de bioéthique contemporaine; chacun devenant du coup bioéthicien de fait. Ce rôle est plus complexe que jamais et, plus que jamais, il ne peut pas être assumé que par un seul individu, aussi expert soit-il. Il est temps de relever le défi qu'indiquait Agich :

It is time for bioethicists to think innovatively about the ethical problems associated with innovation in patient care. To do so, bioethics will need to pay closer attention to the actual clinical, institutional, and professional processes that operate in the development of novel therapeutic interventions (Agich, 2001: 296).

Plus concrètement, une telle instance d'évaluateurs avisés, comme le serait un CECl par exemple, pourrait relever le défi que soulevait Blancquaert et ses collègues (2001: 107) à l'effet que «les décideurs devront se préparer au défi du transfert de technologie [...] et travailler à la construction d'un cadre pour superviser la mise en œuvre des tests et établir des modalités pour leur intégration dans les services cliniques». À cette fin, ajoutent-ils, les méthodes qui favorisent la collecte de données sur la validité analytique et clinique des tests et sur leurs avantages et risques associés devraient faire l'objet d'une évaluation indépendante et multidisciplinaire des données pertinentes, ces évaluations devant être intégrées au processus décisionnel.

Qu'on ne s'y trompe pas, ce défi d'évaluation de nouvelles procédures et de leurs conditions d'intégration dans le processus décisionnel que soulèvent Blancquaert et ses coll. (2001) vise un transfert des connaissances qui soit éthiquement responsable (Legault et coll., 2017).

\section{Conclusion et perspectives}

S'il est de bon ton de dire que l'éthique est une responsabilité partagée, il convient néanmoins d'en assumer les conséquences en outillant efficacement les parties prenantes de nos institutions de recherche et établissements de santé, dont les CER, en vue d'une évaluation éthique plus assurée de ces initiatives menées avec et auprès de populations souvent vulnérables. Ce souci éthique dans le développement et l'adoption de nouvelles pratiques en santé contribuerait à réduire l'un des effets néfastes de cette confusion sur le respect de l'autonomie des personnes et leur droit à consentir. Ces valeurs sociales seraient fragilisées du moment où il serait relativement facile de laisser libre cours à des traitements expérimentaux sur des patients en clinique sous le couvert de soins jugés innovateurs, voire approuvés, selon l'évaluation subjective de chacun. Cette solution, de juger du caractère «recherche» ou «innovateur» selon la seule intention du clinicien, à savoir s'il entend acquérir de nouvelles connaissances ou soigner, bien qu'intéressante, est nettement insuffisante, voire naïve, dans nos contextes de pratique qui valorisent le cumul des rôles professionnels ainsi que la rapidité des applications en clinique des connaissances issues de l'expérimentation humaine. 
Les conflits de rôles, loin d'être rares, sont souvent structurels (Ghaemi and Goodwin, 2007). Des pistes sont donc à explorer dans la foulée de celles proposées dans cet article. D'ici là, la qualité de l'encadrement éthique de certains projets visant de nouvelles pratiques cliniques pourrait s'en trouver sensiblement réduit et les patients pourraient ainsi être exposés à des risques auxquels ils n'auraient pas consenti librement et en toute connaissance de cause.

Plus encore, la notion de traitements, procédures ou soins innovateurs est plus orpheline que jamais, voire abandonnée, depuis la suppression du dernier alinéa de l'ancien article 21 du CCQ en juin 2013 la concernant spécifiquement. En effet, bien que ce retrait règle certains problèmes au plan juridique, il n'en va pas de même au plan éthique. En effet, sur ce dernier plan, le problème reste entier. Le statut innovateur d'une procédure, soin ou traitement, existe bel et bien; car il est une partie intégrante du continuum traversant le développement des connaissances au service des soins, de la recherche à la clinique, et il ne saurait en être autrement. La question de l'encadrement éthique de cette zone d'ombre subsiste néanmoins. Le but ultime de cet article est donc de sensibiliser les communautés concernées, en recherche comme en clinique, à l'importance, voire l'urgence, de répondre aux enjeux éthiques grandissants auxquels la notion de procédures ou soins innovateurs visait précisément à répondre et aux enjeux éthiques en découlant directement, dont ceux liés au respect de l'autonomie de la personne par l'obtention d'un consentement éthiquement valide ainsi que ceux liés à un transfert des connaissances socialement responsable.

\section{Remerciements}

Nous remercions chaleureusement les Instituts de recherche en santé du Canada (IRSC \# 142187) pour leur support à ces travaux, ainsi que Hubert Gagnon et Louise Corbeil pour leurs judicieux conseils. 


\section{Bibliographie}

American College of Physicians. (1998). ACP Ethics Manual (Fourth Edition). ISBN 0-943126-67.

Agich, GJ. (2001). Ethics and innovation in medicine, Journal of Medical Ethics, 27, 295-296

Audy, S. (2006). Le Plan d'action ministériel en éthique de la recherche et en intégrité scientifique : une entreprise insensée? Ministère de la Santé et des Services sociaux. Québec. Sect. 6.2.2. : 253.

Avard, D., Knoppers, M.B. et Lévesque, E. (2004). La génétique et le cadre juridique applicable au secteur de la santé; examens génétiques, recherche en génétique et soins innovateurs, 64 R. du B. 57.

Blancquaert, I. (2000). Availability of Genetic Services: Implementation and Policy Issues, Community Genetics, 3 (4), 179-183.

Blancquaert, I., Cleret de Langavant, G., Bouchard, L., Obadia, A., Chikhaoui, Y. and Battista, R.N. (2001). Oversight Mechanisms for Technology. Transfer in Molecular Genetics. Meeting the Challenge, Isuma, 103-108.

Broekman, M.L., Carrière, M.E. et Bredenoord, A.L. (2016). Surgical innovation: the ethical agenda. A systematic review. Medicine (Baltimore), Vijayaprasad Gopichandran (ed), 95 (25), 1-5.

Conseil d'évaluation des technologies de la santé (CETS). (1991). La transplantation au Québec. Rapport préliminaire sur l'efficacité, les coûts et les caractéristiques organisationnelles. Montréal.

Deleury, E. (2002). Le droit des personnes physiques, Cowansville, Éditions Yvon Blais.

Desjardins, L. (1997). Le consentement à l'expérimentation clinique : Quelles sont les limites de l'acceptable et de l'inacceptable? Journal du Barreau, 29 (4). Repéré à http://www.barreau.qc.ca/pdf/journal/vol29/ no4/consentement.html

Edgar, H. et Rothman, D.J. (1995). The institutional review board and beyond: future challenges to the ethics of human experimentation. Milbank Q., 73 (4), 489-506.

Fonds de la recherche en santé du Québec (FRSQ). (2008; Modifié par le conseil d'administration du FRSQ en décembre 2009). Les Standards du FRSQ sur l'éthique de la recherche en santé humaine et l'intégrité' scientifique. 51 pages.

Fost, N. (1998 Jun). Ethical dilemmas in medical innovation and research: distinguishing experimentation from practice, Semin Perinatol. 22 (3), 223-232.

Gelijns, AC. and Thier, SO. (2002). Medical Innovation and Institutional Interdependence Rethinking University-Industry Connections, Journal of the American Medical Association (JAMA), 287 (1), 72-77.

Ghaemi, S.N. et Goodwin, F.K. (2007). The ethics of clinical innovation in psychopharmacology: Challenging traditional bioethics», Philosophy, Ethics, and Humanities in Medicine, 2 (26) (Open access).

Kerridge, I., Lowe, M. and Henry, D. (1998 Apr). Ethics and evidence based medicine. BMJ., 11 (316[7138]), 1151-1153.

Kouri, R.P. (2013). Observations concernant certains changements apportés au code civil par la loi modifiant le code civil et d'autres dispositions législatives en matière de recherche. Revue de Droit Université de Sherbrooke (RDUS), 43, 867-886.

Kouri, R.P. et Philips-Nootens, S. (1996-97). L' «expérimentation» et les «soins innovateurs» : l'article 21 CCQ. et les affres de l'imprécision. Revue de droit de l'Université de Sherbrooke (R.D.U.S), 27, 89-137, 96. 
Legault, G-A., Verchère, C. and Patenaude, J. (2017 Apr.). Support for the Development of Technological Innovations: Promoting Responsible Social Uses, Science and Engineering Ethics, 1-21. DOI 10.1007/s11948017-9911-5. (Open Access).

Lind, S.E. (1992). Innovative medical therapies: between practice and research. Clinical Research. 36, 546-551.

Moore, F.D. (1969). Therapeutic Innovation: Ethical Boundaries in the Initial Clinical Trials of New Drugs and Surgical Procedures, Daedalus, The MIT Press on behalf of American Academy of Arts \& Sciences, 98 (2), 502522.

Moutel, G., Leroux, N. and Hervé, C. (1998 Apr.). Analysis of a survey of 36 French research committees on intracytoplasmic sperm injection. The Lancet, 351, 1121-1123.

MSSS. Ministère de la santé et des services sociaux. Direction générale de la planification et de l'évaluation. Gouvernement du Québec, (1998, Juin [Dernière modification : 14 avril 1999]).Plan d'action ministériel en éthique de la recherche et en intégrité scientifique. 40 pages.

Parliament UK., 2013. Medical Innovation (No.2) Bill 2013-14. Repéré à http://services. parliament.uk/ bills/2013-14/medicalinnovationno2.html

Patenaude, J. et Cabanac, J. (2000). Quelle recherche, quelle évaluation? Le cas de la distinction entre soin expérimental et soin innovateur. Éthique Publique, 2, 89-98.

Patenaude, J., Grant, A., Xhignesse, M., Leblanc, F. et Courteau, J., (2008 Jan). Evaluation of Clinical Innovation: A Gray Zone in the Ethics of Modern Clinical Practice? Journal of General Internal Medicine (JGIM), 23 (Suppl 1), 27-31.

Prentice, E.D., Gordon B.G. and Lin M.H. (1997). Determining when a clinical activity should be classified as research requiring institutional review board review. The Journal of Extra-Corporeal Technology, 29 (2), 88-91.

Sackett, D.L. (1997). Evidence-based medicine. Dans Zipursky, A. (dir.). Seminars in Perinatology. Fatal and Neonatal Hematology for the 21st Century. 21 (1), 3-5.

Tonelli, M.R., (Dec1998). The philosophical limits of evidence-based medicine. Academic Medicine, 73 (12), 1234-1240.

Viens, E-J. (2015). Le consentement des personnes vulnérables à la recherche: regard sur les amendements et les omissions de l'article 21 du code civil du Québec. Mémoire de maîtrise en droit. Faculté de droit. Université de Sherbrooke.

Wheeler, R. 2000, 3 June. «One person's innovation is another's experiment». BMJ. 320 : 1548.

Weisstub, D.N. et Verdun-Jones, S. (1996-97). «Pour une distinction entre l'expérimentation thérapeutique et l'expérimentation non thérapeutique». Revue de Droit Université de Sherbrooke (R.D.U.S.), 27, 49-87.

Wilfond, B.S. (1995 May-June). Screening policy for cystic fibrosis: the role of evidence. Hastings Center Report, S21-3. 